\title{
ASS and SULT2A1 are Novel and Sensitive Biomarkers of Acute Hepatic Injury-A Comparative Study in Animal Models
}

\author{
Victor Prima ${ }^{1 *}$, Mengde $\mathrm{Cao}^{1}$ and Stanislav I Svetlov ${ }^{1,2 *}$
}

${ }^{1}$ Banyan Laboratories, Inc., Alachua, FL, USA

${ }^{2}$ Department of Medicine, University of Florida, Gainesville, FL, USA

\begin{abstract}
Liver and kidney damage associated with polytrauma, endotoxic shock/sepsis, and organ transplantation, are among the leading causes of the multiple organ failure. Development of novel sensitive biomarkers that detect early stages of liver and kidney injury is vital for the effective diagnostics and treatment of these life-threatening conditions. Previously, we identified several hepatic proteins, including Argininosuccinate Synthase (ASS) and sulfotransferases which were degraded in the liver and rapidly released into circulation during Ischemia/Reperfusion $(\mathrm{I} / \mathrm{R})$ injury. Here we compared sensitivity and specificity of the newly developed sandwich ELISA assays for ASS and the sulfotransferase isoform SULT2A1 with the standard clinical liver and kidney tests Alanine Aminotransferase (ALT) and Aspartate Transaminase (AST) in various pre-clinical models of acute injury. Our data suggest that ASS and SULT2A1 have superior characteristics for liver and kidney health assessment in endotoxemia, Ischemia/ Reperfusion (I/R), chemical and drug-induced liver injury and may be of high potential value for clinical applications.
\end{abstract}

Keywords: Biomarkers; Liver; ASS; SULT2A1; Sepsis; Toxicity; Shock

\section{Introduction}

Liver and kidney damage and failure due to various forms of intoxication and abdominal injury are significant sources of overall morbidity and mortality in the US and worldwide. Persistent hepatic injury occurs during viral hepatitis, fatty liver disease (steatohepatitis), drug or alcohol and autoimmune induced hepatitis. Clinical conditions are numerous where related hepatic and renal injuries are critical components of multi-organ failure caused by complex trauma including blast injury, septic shock, and graft failure after liver transplantation often leading to death of the patient. Specific biochemical markers have become mandatory in diagnosing dysfunction for a number of organs, for example myocardial infarction or hepatitis. The increase of ALT and AST in blood has been used in clinical practice for a long time for diagnostics of viral hepatitis of all types and alcoholic/toxic hepatitis, and monitoring of treatment. Several other enzymes such as isocitrate dehydrogenase [1] and anti-oxidative enzyme GlutathioneS-Transferase (GST) [2] were also shown as potential markers for viral hepatitis injury. $\gamma$-Glutamyl Transferase $(\gamma-G T)$ has been used for diagnostics of hepatitis accompanied by cholestasis and hepatobiliary injury and viral hepatitis B and C [3,4]. Elevated GST levels in blood were detected in posttraumatic hepatic injury in primates, acute hepatotoxicity, and intestinal ischemia in rats [5-7]. However, diagnostic tests of toxic hepatitis or alcoholic liver disease reflect advanced stages of liver diseases with profound levels of hepatocellular death, and do not allow to distinguish between parenchymal and hepatic endothelial injury and/or to determine magnitude and reversibility of damage. Thus, a clinical need is apparent for specific, non-invasive pathogenically relevant biomarkers which would diagnose the magnitude and phase of liver injury for better diagnostics and control of treatment. We set out to develop biomarkers for liver focusing on molecules specific to the liver, which could be substrates for proteolytic cleavage. By using liver proteomic degradomics approach we identified several biomarker candidates, which were then tested in experimental liver I/R injury in rats [8]. The most promising molecules have been Argininosuccinate Synthase (ASS) and Estrogen Sulfotransferase (EST-1). We have found that hepatic ASS is subjected to proteolytic cleavage in the liver via pro-apoptotic caspase-3, which is activated upon hepatic toxic, ischemic or viral insult, and can be released in blood [8]. Subsequently, we discovered that SULT2A1 isoform of sulfotransferase is a more sensitive in detecting liver injury. Also, SULT2A1 possess a slightly greater value regarding specificity and assay development. In the current study we compared sensitivity and specificity of the newly developed ELISA assays for ASS and the SULT2A1 with standard clinical liver and kidney function assays for Alanine Aminotransferase (ALT) and Aspartate Transaminase (AST) in various pre-clinical models of acute toxicity. Our data suggest that ASS and SULT2A1 have superior characteristics for liver and kidney health assessment in endotoxemia, Ischemia/Reperfusion (I/R), chemical and drug-induced liver and kidney injury and may be of high potential value for clinical applications.

\section{Materials and Methods}

\section{Reagents}

Lipopolysaccharide (LPS) from Escherichia coli O111:B4, D-Galactosamine (D-Gal) and Carbon tetrachloride $\left(\mathrm{CCl}_{4}\right)$ were purchased from Sigma (St. Louis, MO, USA). The levels of endogenous ASS and SULT2A1 in serum were determined by SW ELISA assays (Banyan Biomarkers, Inc.) using rabbit polyclonal antibodies as capture and mouse monoclonal as detection antibodies. Color development was accomplished using anti-mouse HRP- conjugated antibodies

*Corresponding authors: Stanislav I Svetlov, Banyan Laboratories, Inc. 12085 Research Drive, Alachua, FL 32615, USA, Tel: (386) 518-6756; E-mail: ssvetlov@banyanbio.com

Victor Prima, Department of Medicine, University of Florida, Gainesville, FL, USA, Tel: (386) 518-6761; E-mail: vprima@banyanbio.com

Received November 27, 2012; Accepted January 05, 2013; Published January 10,2013

Citation: Prima V, Cao M, Svetlov S (2013) ASS and SULT2A1 are Novel and Sensitive Biomarkers of Acute Hepatic Injury-A Comparative Study in Animal Models. J Liver 2: 115. doi:10.4172/2167-0889.1000115

Copyright: (c) 2013 Prima V, et al. This is an open-access article distributed unde the terms of the Creative Commons Attribution License, which permits unrestricted use, distribution, and reproduction in any medium, provided the original author and source are credited. 
followed by TMB substrate incubation. ASS and SULT2A1 levels were calculated from a calibration curve using human recombinant proteins prepared according [9] as standards.

\section{Transaminase activities}

The alanine aminotransferase (ALT, C.E.2.6.1.2) and aspartate aminotransferase (AST, C.E.2.6.1.1) activities in animal blood were measured using end-point colorimetric tests (BioVision, Milpitas, CA) in duplicate according to the manufacturer's instructions. .

\section{Animals}

Adult male Sprague-Dawley rats (200-225 g) and Balb/c mice (19$22 \mathrm{~g}$ ) (Harlan Laboratories Inc., Indianapolis, IN) were used in this study. The animals were housed under constant temperature $\left(22^{\circ} \mathrm{C}\right)$ and humidity with $12 \mathrm{~h}$ light/dark cycle and had access to chow and water as much as desired throughout the study. All experiments were performed in adherence with the National Institutes of Health guidelines for the use of experimental animals and were approved by the Institutional Animal Care and Use Committee of the University of Florida.

\section{Rodent endotoxemia models}

LPS/D-galactosamine acute liver injury: Lipopolysaccharide from E. coli (LPS, $10 \mu \mathrm{g} / \mathrm{kg}$ ) plus D-galactosamine (D-Gal, $500 \mathrm{mg} / \mathrm{kg}$ ), or LPS alone $(100 \mu \mathrm{g} / \mathrm{kg})$ or saline were injected intraperitoneally (i.p.) in Sprague-Dawley rats as described previously [10,11]. Blood was collected from heart of anesthetized animals at terminal time points 1 $\mathrm{h}, 2 \mathrm{~h}, 3 \mathrm{~h}$ and $24 \mathrm{~h}$ after the treatment, using at least 3 different rats for each time point.

Mouse endotoxemia model: For biomarker release studies Balb/c mice were given either i.p. E. coli LPS (15 mg/kg) alone or LPS followed in $1 \mathrm{~h}$ by injection with $\mathrm{rASS}(5 \mathrm{mg} / \mathrm{kg})$.

\section{Rat chemical hepatotoxicity models}

Carbon tetrachloride $\left(\mathrm{CCl}_{4}\right.$, diluted to $0.25-0.5 \mathrm{ml} / \mathrm{kg}$ in $0.2 \mathrm{ml}$ olive oil) was injected as a bolus intraperitoneally (i.p.) in SpragueDawley rats. Blood was collected from heart of anesthetized animals at terminal time points $1 \mathrm{~h}, 6 \mathrm{~h}$ and $24 \mathrm{~h}$ after the treatment, using at least 3 different rats for each time point.

\section{Rat model of liver ischemia/reperfusion injury}

Adult male Sprague-Dawley rats (220-250 g) were anesthetized with $4 \%$ Isoflurane for $4 \mathrm{~min}$ in a chamber until a surgical level of anesthesia was achieved. Animals were placed on the heating pad and delivery of anesthetic gas continued via a nose cone throughout the surgery. A midline approximately 3 -cm-long laparotomy was made, and the liver was exposed. The portal triad was exposed and occluded for $30 \mathrm{~min}$ using an atraumatic vascular clamp. After $30 \mathrm{~min}$ of normothermic ischemia, recirculation of the blood through the ischemic liver was achieved by removing the clamp for additional 10, $30 \mathrm{~min}, 1$ and $3 \mathrm{~h}$. At the end of reperfusion, blood was collected from heart; the liver was briefly perfused with cold Phosphate-Buffered Saline (PBS) to remove residual blood and taken for analysis. For comparison unilateral kidney I/R was performed in a similar manner as for liver with a renal artery and a vein dissected and occluded for $30 \mathrm{~min}$. After 30 min of normothermic ischemia, recirculation of the blood through the ischemic kidney was restored for $30 \mathrm{~min}$.

\section{Statistics}

Statistical analyses were performed using GraphPad Prism 5 software. Data were evaluated by 2 -tailed unpaired t-test. The criterion for statistical significance was set at $\mathrm{p}<0.05$ or $\mathrm{p}<0.01$.

\section{Results}

\section{ASS and SULT2A1 are sensitive biomarkers of endotoxicity/ liver injury}

To assess quantitatively the levels of endogenous ASS and in rat serum after LPS treatment alone and in combination with the liver injury priming agent D-Galactosamine (D-Gal) we used the ELISA assay developed at Banyan Biomarkers [9]. As seen in figure 1A, ASS significantly accumulated in serum within one $\mathrm{h}$ after i.p. injection of LPS $(100 \mu \mathrm{g} / \mathrm{kg})$, attained a maximum increase of $\sim 25$-fold over baseline at $2 \mathrm{~h}$, and decreased but remained significantly elevated at 3 and $24 \mathrm{~h}$. In contrast to ASS, serum ALT levels after injection of $100 \mu \mathrm{g} / \mathrm{kg}$ LPS alone did not change significantly (Figure 1B), serum AST levels changed significantly only $3 \mathrm{~h}$ after LPS injection (Figure 1B) and no pathomorphological injury to the liver was observed at $24 \mathrm{~h}$ after injection (data not shown). When rats $(\mathrm{n}=8)$ were treated with lower doses of LPS $(10 \mu \mathrm{g} / \mathrm{kg})$ together with D-Gal to sensitize liver to LPS exposure, serum ASS levels raised significantly at $1 \mathrm{~h}$ and achieved nearly 1000-fold levels over control (saline-treated) in the animals which were alive but exhibited strong signs of terminal illness (2 out of 8) (Figure 1D). For this group corresponding ALT/AST levels were 29/12-fold over the control level (Figures $1 \mathrm{E}$ and $1 \mathrm{~F}$ ). In rats recovered from treatment ( 3 out of 8 ), serum ASS levels declined to nearly baseline after $24 \mathrm{~h}$, but were elevated over control rats (Figure 1D). Serum ALT activity was found to increase significantly at $2 \mathrm{~h}$ and was substantially elevated at $24 \mathrm{~h}$ after LPS/D-Gal injection compared to control values (Figure 1E). Serum AST levels changed significantly compared to control only $3 \mathrm{~h}$ after LPS/D-Gal injection (Figure 1F). The data shown above are in concordance with more prominent ASS accumulation in blood observed after i.p. injection of much higher doses of bacterial LPS in mice $(15 \mathrm{mg} / \mathrm{kg}$ ) (Figure 2A). It is worth noting that i.p. administered rASS is cleared from circulation over an $18 \mathrm{~h}$ time period in the absence of liver injury (Figure $2 \mathrm{~B}$ ) so that the LPS-related elevation at $18 \mathrm{~h}$ (Figure $2 \mathrm{~A}$ ) exceeds the normal clearance kinetics. SULT2A1 ELISA developed at Banyan Biomarkers also has high sensitivity for LPS intoxication. Following $24 \mathrm{~h}$ after i.p. injection of LPS only (Figure 6B) SULT2A1 serum level was 200 -fold over control level.

\section{ASS is a sensitive liver and kidney I/R injury biomarker}

We assessed ASS in blood of rats subjected to liver Ischemia/ Reperfusion (I/R) injury on different reperfusion time with a fixed $30 \mathrm{~min}$ of ischemia period. As figure 3 indicates, the magnitude of $\mathrm{I} / \mathrm{R}$ injury depends largely on reperfusion time. Massive release and accumulation of ASS in blood was detected immediately after complete $30 \mathrm{~min}$ ischemia (0 reperfusion time), was reperfusion time-dependent and rapidly attained a steady state within $30 \mathrm{~min}$, and persisted up until $180 \mathrm{~min}$ after initiation of reperfusion. Blood ASS levels correlated strongly with the severity of liver injury determined by histopathology of liver tissue (data not shown). As can be seen, ASS increase over sham treated animals was 23 -fold immediately after ischemia as compared to ALT/AST which did not elevate at this time. Moreover, the magnitude of ASS increase has been 100-300-fold throughout $3 \mathrm{~h}$ reperfusion, whereas ALT/AST increase was 7 to 8 -fold over sham-operated rats. The results obtained in unilateral kidney $\mathrm{I} / \mathrm{R}$ studies show a sharp ( $\sim$-fold) increase of ASS concentration in rat blood after $30 \mathrm{~min}$ of normothermic ischemia followed by 30 min reperfusion (Figure $4 \mathrm{~A}$ ). 

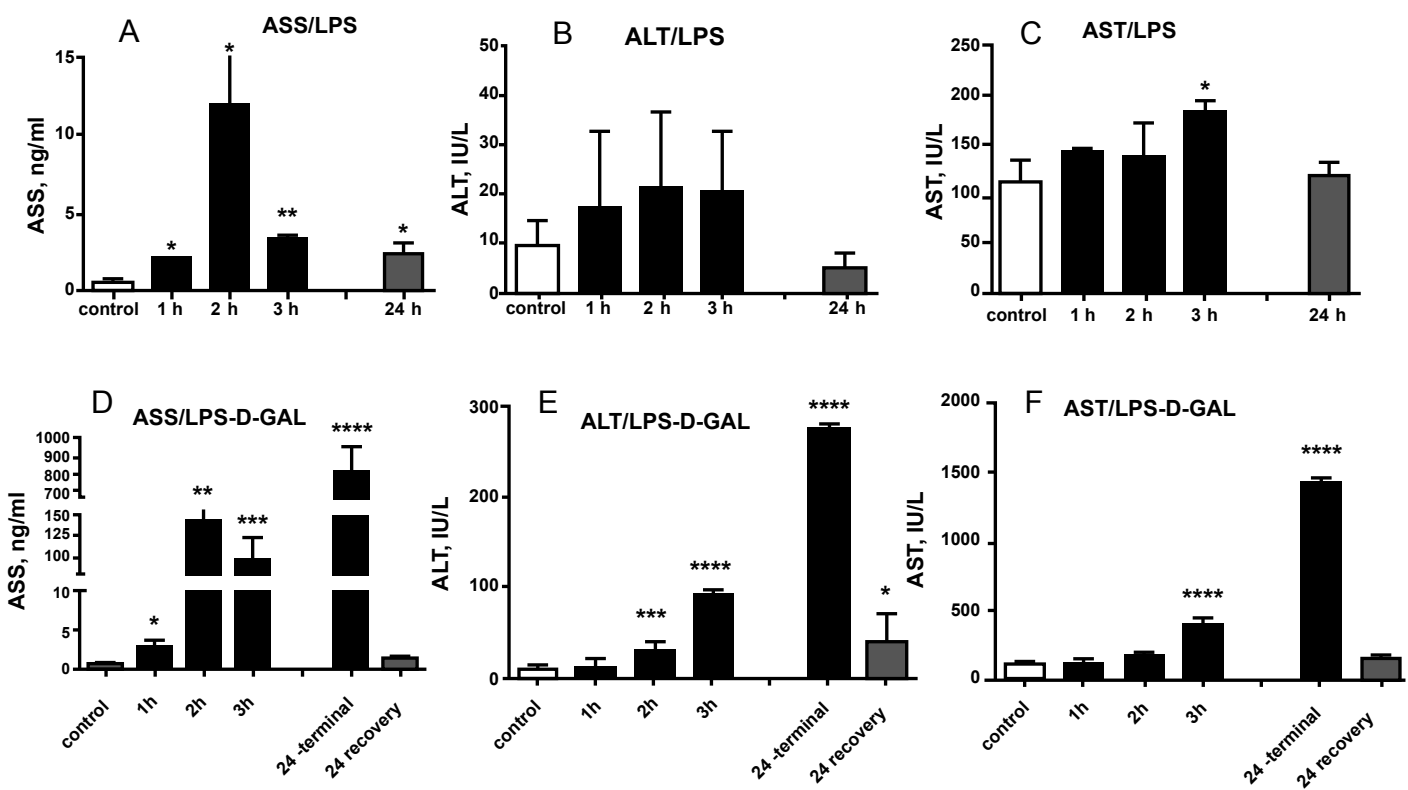

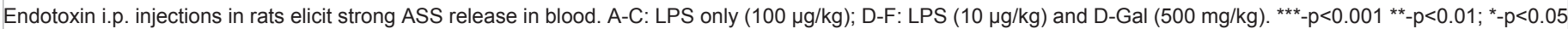
vs. control samples.

Figure 1: ASS is a potential biomarker of endotoxicity/liver injury.

No significant changes were observed in blood ALT (Figure 4B) and AST (Figure 4C) activities.

\section{ASS and SULT2A1 are potential biomarkers of chemical hepatotoxicity}

ASS rapidly accumulated in plasma $1 \mathrm{~h}$ after $\mathrm{CCl}_{4}$ injection $(\sim 15-$ fold over control) and further increased at $24 \mathrm{~h}$ post-treatment $(>50$ fold over control) (Figure 5A). No significant increase in plasma ALT was detected at $1 \mathrm{~h}$ following injection; ALT was elevated at $24 \mathrm{~h}$ posttreatment 6-fold over control (Figure 5B). SULT2A1 level in serum also surged $1 \mathrm{~h}$ after $\mathrm{CCl}_{4}$ injection ( 19-fold over control) and remained significantly elevated up to $24 \mathrm{~h}$ post-treatment (Figure 6A).

\section{Discussion}

In the previous studies we employed liver proteomic degradomics approach and identified several biomarker candidates, which were then preliminarily validated in experimental liver I/R injury and LPS/DGal injury in rats [8]. LPS/D-Gal treatment is an established model of targeted LPS-dependent liver injury accompanied by a massive hepatic apoptosis and release of amino transferases ALT and AST [12,13]. As seen on figure 1, rat liver injury induced by injection of bacterial LPS only (Figure 1A) or LPS and D-Gal combination (Figure 1D) was accompanied by a fast rise of serum ASS levels. At the same time-points changes of ALT (Figures $1 \mathrm{~B}$ and $1 \mathrm{E}$ ) and AST (Figures $1 \mathrm{C}$ and $1 \mathrm{~F}$ ) activities were far less pronounced if significant at all.

Our assessments of the ASS utility in the rat model of liver ischemia/ reperfusion injury demonstrate high correlation of ASS levels in serum with duration of reperfusion and magnitude of liver injury (Figure $3 \mathrm{~A})$. Sensitivity on the early stages of liver injury and dynamic range of ASS ELISA far exceeds that of ALT/AST (Figures 3B and 3C). Strong ASS response to the kidney damage was found also in the rat model of kidney ischemia/reperfusion (Figure 4A). Again, here ASS ELISA was a better injury indicator than ALT/AST assays (Figures $4 \mathrm{~B}$ and
4C). Exposure to high concentrations of toxic chemical compounds or drugs overused for medical or recreational purposes can cause liver and kidney damage [14-17]. Therefore important diagnostic need can be met with ELISA tests for ASS and SULT2A1 employed as illustrated for $\mathrm{CCl}_{4}$ (Figures 5A and 6A). Time/dose-dependent accumulation of ASS and SULT2A1 in blood strongly correlates with the toxicity levels achieved.

The use of ASS and SULT2A1 as components of biomarker panel for liver/kidney injury confers a number of advantages over existing 'surrogate' biomarkers: (i) ASS and SULT2A1 accumulated in blood earlier than ALT/AST (Figures 1,3,5 and 6), (ii) the diagnostic window was much larger with the fold increase ranged from 2 to 1000 -fold depending on the injury magnitude (Figures 1 and 3-6), (iii) ASS and SULT2A1 declined faster than ALT/AST upon resolution of damage (Figure 1), and (iv) ASS/ SULT2A1 play roles in pathogenesis of hepatic injury linking oxidative stress, liver function (ASS), and responses to toxic insults (SULT2A1). ASS is not found in erythrocytes or other blood cells. Thus, assessments of ASS and SULT2A1 in serum or plasma are not confounded by red blood cell hemolysis; unlike ALT or AST, both serum and plasma can be used for analysis. Ideally, whole blood can be employed in the future express diagnostics of ischemic liver damage, for example in doctor offices, or during emergency combat operations. Recently, the potential diagnostic value for liver type Arginase-I (Arg-I) and Carbamoyl Phosphate Synthetase-1 (CPS1) was reported in rat liver ischemia/reperfusion [18-20]. While Arg-I and CPS-1 appear to be promising candidates as biomarkers for liver injury, the comprehensive studies of these enzymes have not been performed.

Our selection of liver biomarkers followed several criteria that a potential marker needs to fulfill for initiation of assay development: specificity of the marker to the organ of interest (tissue panel and protein expression level), comprehensive protein analysis for stability, 


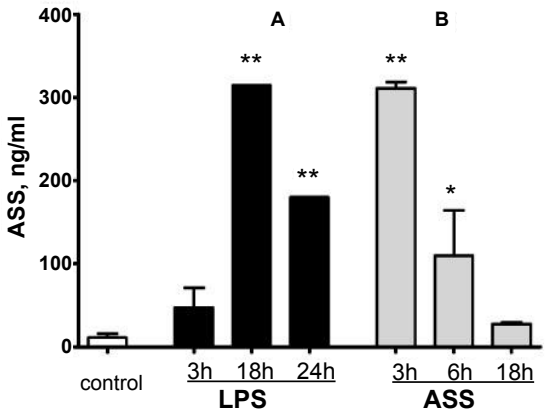

A: Mice serum ASS levels induced by i.p. LPS $(15 \mathrm{mg} / \mathrm{kg}$ ) injection. B: Serum ASS kinetics following i.p. injection with $\mathrm{rASS}(5 \mathrm{mg} / \mathrm{kg})$. Mean+S.D. and T-test analysis are shown. ${ }^{* *}-p<0.01 ;{ }^{*}-p<0.05$ vs. control samples.

Figure 2: Serum ASS accumulates following LPS administration in mice.

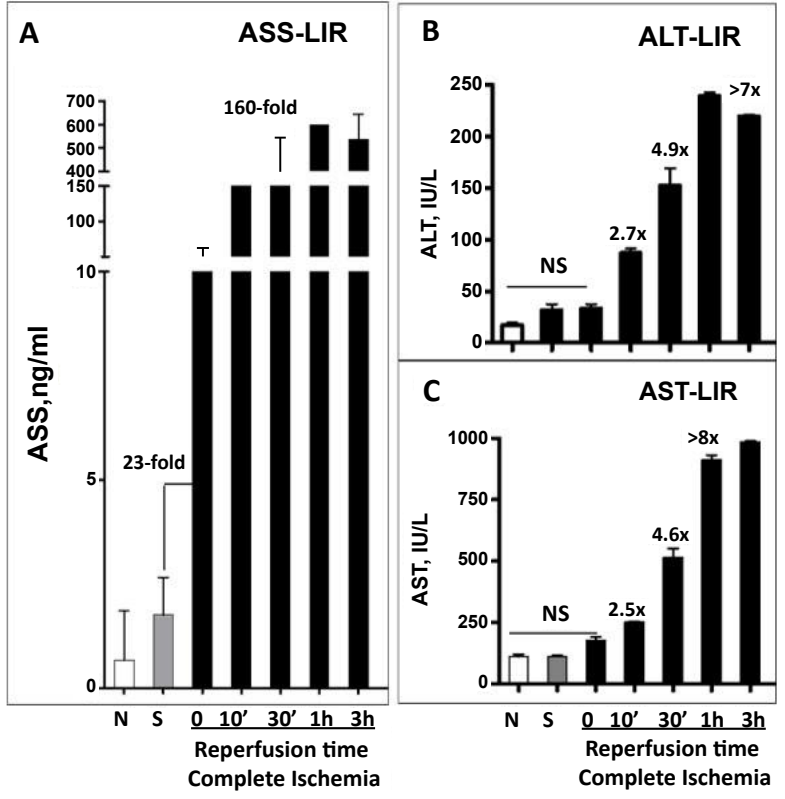

A: ASS accumulation in blood; B and C: ALT and AST enzymatic assays in the same samples; N-naive $(n=5)$; S-sham operated rats $(n=4) ; 0$ to 3 hours reperfusion (4-6 rats), 24 hours after partial reperfusion $(n=4)$. ALT and AST were measured using end-point colorimetric tests in duplicate Mean+S.D and T-test analysis are shown.

Figure 3: Liver injury markers correlate with I/R injury/reperfusion time.

antigenicity and isoform similarity. Ideally, biomarkers should employ biological substrates unique to the organ and, at the same time, provide information on injury mechanisms, a criterion that is used to distinguish biochemical markers from surrogate markers of injury since surrogate markers usually do not provide information on injury mechanisms. SULT2A1 and ASS fit criteria for biochemical markers: they are closely involved in major protective systems including liver and kidney. SULT2A1 represents detoxification function of liver [21]. In the liver, SULT2A1 plays an important role in bile acid homeostasis and protection against the toxic effects of bile acids. SULT2A1 is also found at minor levels in jejunum, ileum, cecum and kidney cytosol samples. Argininosuccinate Synthase (ASS) is a link between urea cycle and Nitric Oxide (NO) synthesis ([22]) which plays a major role in responses to ischemia, oxidative stress and toxins upon activation of inducible NO synthase in hepatocytes. In hepatic endothelial cells, constitutively expressed eNO synthase can utilize arginine supplied by consecutive actions of ASS and ASL and thereby provide the initial responses to injurious agents. As our prior experiments showed, if the endotoxin injection was followed by that of recombinant ASS, it significantly alleviated systemic septic symptoms and inflammation compared to untreated endotoxemia substantiating a hypothesis that systemic ASS release is an innate immune system response to endotoxic shock [9]. It should be noted that ASS is now recognized as a ubiquitous enzyme in mammalian tissues with the highest values
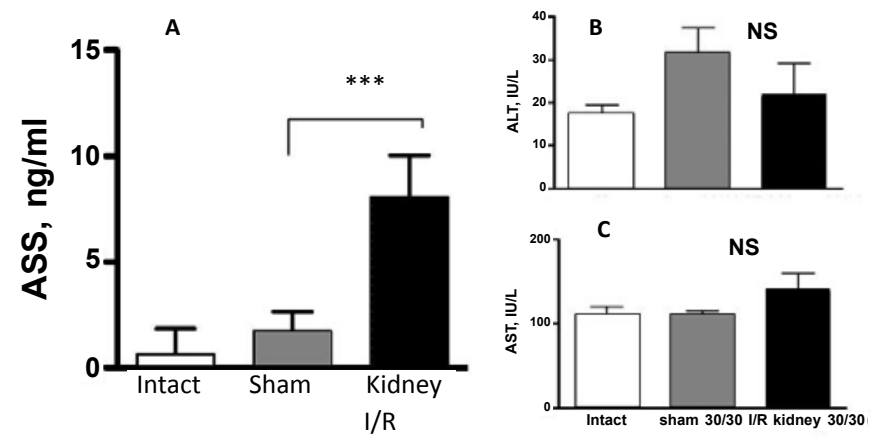

Unilateral kidney Ischemia-Reperfusion. A: ASS ELISA; B: ALT; C: AST. For details please see Materials and Methods. Mean+S.D. and T-test analysis are shown. ${ }^{* * *}-p<0.001$ vs. control samples.

Figure 4: ASS accumulates in serum as a response to kidney I/R injury.
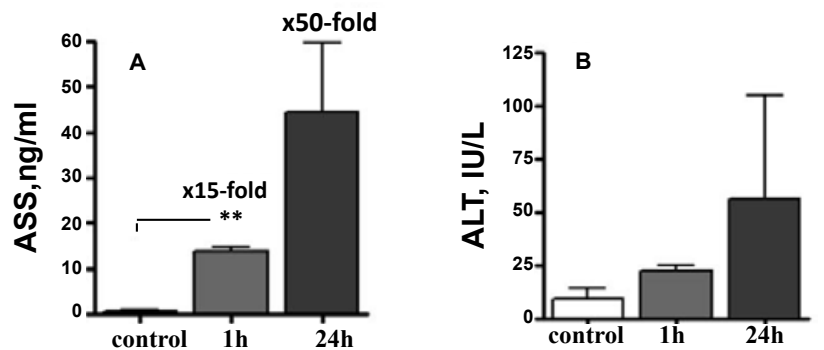

Plasma ASS accumulation after CCl4 i.p. injection $(0.5 \mathrm{ml} / \mathrm{kg}$ in $0.2 \mathrm{ml}$ veg. oil). A: ASS ELISA; B: ALT assay. For details please see Materials and Methods. Mean+S.D. and T-test analysis are shown. ${ }^{* *}-p<0.01 ;{ }^{*}-p<0.05$ vs. control samples.

Figure 5: Serum ASS elevation reflecting chemical hepatotoxicity.
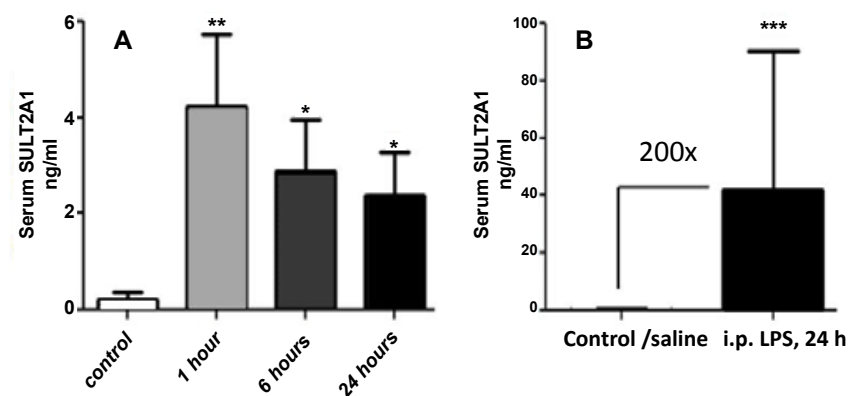

A: Time-course of serum levels of SULT2A1 after i.p. injection of $0.25 \mathrm{ml} / \mathrm{kg}$ of carbon tetrachloride in olive oil. B: SULT2A1 24 hours after i.p. injection of LPS $\left(20 \mathrm{mg} / \mathrm{kg}\right.$ ). Control: solvent only. Mean+S.D. values are shown. ${ }^{* *}-p<0.01$; ${ }^{*}$ - $p<0.05$ vs. control samples.

Figure 6: SULT2A1 accumulates in serum after rat treatment with $\mathrm{CCl} 4$ or LPS 
Citation: Prima V, Cao M, Svetlov S (2013) ASS and SULT2A1 are Novel and Sensitive Biomarkers of Acute Hepatic Injury-A Comparative Study in Animal Models. J Liver 2: 115. doi:10.4172/2167-0889.1000115

Page 5 of 5

(both mRNA and protein levels) found in the liver and kidneys [23]. Nevertheless, because liver is the largest rat organ [24], we consider it as the primary source of endogenous ASS in circulation even accounting for the possible input from non-hepatic tissues [25].

\section{Conclusion}

Our data suggest that ASS and SULT2A1 have superior characteristics over traditional biomarkers for liver and kidney health assessment in endotoxemia, I/R, chemical and drug-induced liver injury and may be of high potential value for clinical applications.

\section{Acknowledgments}

The authors thank Ms. Olena Glushakova and Ms. Sabrina Singleton for their expert technical assistance. This work was supported by the National Institutes of Health (NIH) grant [5R44DK074205] and NIH Recovery Act Administrative Summer Supplement [R44DK074205-03S1].

\section{References}

1. Chung YH, Jung SA, Song BC, Chang WY, Kim JA, et al. (2001) Plasma isocitrate dehydrogenase as a marker of centrilobular hepatic necrosis in patients with hyperthyroidism. J Clin Gastroenterol 33: 118-122.

2. Thorburn D, Bird GL, Spence E, MacSween RN, Mills PR (1996) alphaGlutathione S-transferase levels in chronic hepatitis C infection and the effect of alpha-interferon therapy. Clin Chim Acta 253: 171-180.

3. Colombatto P, Randone A, Civitico G, Monti Gorin J, Dolci L, et al. (1996) Hepatitis $G$ virus RNA in the serum of patients with elevated gamma glutamyl transpeptidase and alkaline phosphatase: a specific liver disease? [corrected]. J Viral Hepat 3: 301-306.

4. Reynaud M, Schellenberg F, Loisequx-Meunier MN, Schwan R, Maradeix B, et al. (2000) Objective diagnosis of alcohol abuse: compared values of carbohydrate-deficient transferrin (CDT), gamma-glutamyl transferase (GGT), and mean corpuscular volume (MCV). Alcohol Clin Exp Res 24: 1414-1419.

5. Giffen PS, Pick CR, Price MA, Williams A, York MJ (2002) Alpha-glutathione S-transferase in the assessment of hepatotoxicity--its diagnostic utility in comparison with other recognized markers in the Wistar Han rat. Toxicol Pathol 30: 365-372.

6. Khurana S, Corbally MT, Manning F, Armenise T, Kierce B, et al. (2002) Glutathione S-transferase: a potential new marker of intestinal ischemia. J Pediatr Surg 37: 1543-1548.

7. Redl H, Schlag G, Paul E, Davies J (1995) Plasma glutathione S-transferase as an early marker of posttraumatic hepatic injury in non-human primates. Shock 3: 395-397.

8. Svetlov SI, Xiang Y, Oli MW, Foley DP, Huang G, et al. (2006) Identification and preliminary validation of novel biomarkers of acute hepatic ischaemia/ reperfusion injury using dual-platform proteomic/degradomic approaches. Biomarkers 11: 355-369.

9. Prima V, Wang A, Molina G, Wang KK, Svetlov SI (2011) Inhibition of LPS toxicity by hepatic argininosuccinate synthase (ASS): novel roles for ASS in innate immune responses to bacterial infection. Int Immunopharmacol 11: 1180-1188.
10. Jones JJ, Fan J, Nathens AB, Kapus A, Shekhman M, et al. (1999) Redox manipulation using the thiol-oxidizing agent diethyl maleate prevents hepatocellular necrosis and apoptosis in a rodent endotoxemia model Hepatology 30: 714-724.

11. Dokladny K, Kozak A, Wachulec M, Wallen ES, Menache MG, et al. (2001) Effect of heat stress on LPS-induced febrile response in D-galactosaminesensitized rats. Am J Physiol Regul Integr Comp Physiol 280: R338-R344.

12. Zanobbio L, Palazzo M, Gariboldi S, Dusio GF, Cardani D, et al. (2009) Intestinal glucose uptake protects liver from lipopolysaccharide and D-galactosamine acetaminophen, and alpha-amanitin in mice. Am J Pathol 175: 1066-1076.

13. Cho JY, Yeon JD, Kim JY, Yoo ES, Yu YH, et al. (2000) Hepatoprotection by human epidermal growth factor (hEGF) against experimental hepatitis induced by D-galactosamine (D-galN) or D-GalN/lipopolysaccharide. Biol Pharm Bull 23: $1243-1246$.

14. Recknagel RO, Glende EA Jr, Dolak JA, Waller RL (1989) Mechanisms of carbon tetrachloride toxicity. Pharmacol Ther 43:139-154.

15. Jones AL, Simpson KJ (1999) Review article: mechanisms and management of hepatotoxicity in ecstasy (MDMA) and amphetamine intoxications. Aliment Pharmacol Ther 13: 129-133.

16. Schreiner GE (1972) Toxic nephropathy due to drugs, solvents and metals Prog Biochem Pharmacol 7: 248-284.

17. Rood AS, McGavran PD, Aanenson JW, Till JE (2001) Stochastic estimates of exposure and cancer risk from carbon tetrachloride released to the air from the rocky flats plant. Risk Anal 21: 675-695

18. Ikemoto M, Tsunekawa S, Toda Y, Totani M (2001) Liver-type arginase is a highly sensitive marker for hepatocellular damage in rats. Clin Chem 47: 946948.

19. Langle F, Roth E, Steininger R, Winkler S, Mühlbacher F (1995) Arginase release following liver reperfusion. Evidence of hemodynamic action of arginase infusions. Transplantation 59: 1542-1549.

20. Ozaki M, Terada K, Kanazawa M, Fujiyama S, Tomita K, et al. (1994) Enzymelinked immunosorbent assay of carbamoylphosphate synthetase I: plasma enzyme in rat experimental hepatitis and its clearance. Enzyme Protein 48 213-221.

21. Glatt H, Boeing H, Engelke CE, Ma L, Kuhlow A, et al. (2001) Human cytosolic sulphotransferases: genetics, characteristics, toxicological aspects. Mutat Res 482: $27-40$.

22. Haines RJ, Pendleton LC, Eichler DC (2011) Argininosuccinate synthase: at the center of arginine metabolism. Int J Biochem Mol Biol 2: 8-23.

23. Husson A, Brasse-Lagnel C, Fairand A, Renouf S, Lavoinne A (2003) Argininosuccinate synthetase from the urea cycle to the citrulline-NO cycle. Eur J Biochem 270: 1887-1899.

24. Schoeffner DJ, Warren DA, Muralidara S, Bruckner JV, Simmons JE (1999) Organ weights and fat volume in rats as a function of strain and age. J Toxicol Environ Health A 56: 449-462.

25. Nagasaki A, Gotoh T, Takeya M, Yu Y, Takiguchi M, et al. (1996) Coinduction of nitric oxide synthase, argininosuccinate synthetase, and argininosuccinate lyase in lipopolysaccharide-treated rats. RNA blot, immunoblot, and immunohistochemical analyses. J Biol Chem 271: 2658-2662. 